\title{
Prevalencia de caries dental y factores familiares en niños escolares de Cartagena de Indias, Colombia
}

The prevalence of dental caries related to family factors in schoolchildren from the city of Cartagena in Colombia

Shyrley Díaz-Cárdenas y Farith González-Martínez

Departamento de Odontología Preventiva y Social, Facultad de Odontología Universidad de Cartagena.shyrley77@hotmail.com, farithgm@hotmail.com

Recibido 19 Febrero 2010/Enviado para Modificación 28 Noviembre 2010/Aceptado 10 Diciembre 2010

\section{RESUMEN}

Objetivo Describir la prevalencia y severidad de la caries en niños del colegio John F. Kennedy de Cartagena de Indias y su relación con factores familiares.

Métodos Estudio descriptivo transversal, en 243 estudiantes. Se evaluó la prevalencia de caries dental mediante el índice COP-D y ceo-d, la severidad mediante los criterios ICDAS II 2005 y para las variables familiares se diligenció un cuestionario que incluía el APGAR familiar para medir funcionalidad familiar. Se realizó el análisis mediante estadísticas descriptivas y la significancia de las relaciones entre variables se evaluaron mediante la prueba $\chi^{2}$.

Resultados La prevalencia de caries fue de $51 \%$ (IC $95 \%$; 45-59), el $38 \%$ (IC $95 \%$; 31 44) de los padres habían accedido a la secundaria incompleta, el $44 \%($ IC 95 \%;39-54) vivían en unión libre, $47 \%$ (IC $95 \%$; 40-53) en estrato socioeconómico bajo, el 53 \% (IC $95 \% ; 47-57$ ) en familias nucleares, $47 \%$ (IC $95 \% ; 41-53$ ) reciben ingresos menores a un salario mínimo, $66 \%$ (IC $95 \%$; 58-70) afiliadas al sistema de salud y el $59 \%$ (IC $95 \%$ : 52-66) viven en familias disfuncionales. A través del análisis bivariado solo se encontró significancia estadística al relacionar el nivel de escolaridad de secundaria incompleta del padre con la presencia de caries dental $(p=0,04)$.

Conclusiones La mayoría de las variables si bien no mostraron significancia estadística para explicar la presencia de la caries dental, aportan indicadores de importancia epidemiológica, lo cual permitiría tomar decisiones desde la salud pública.

Palabras Clave: Caries dental, medicina familiar y comunitaria, preescolar (fuente: Decs, BIREME).

\section{ABSTRACT}

Objective Describing the prevalence and severity of caries in schoolchildren attending the John F. Kennedy school in Cartagena and its relationship to family factors.

Methods This was a cross-sectional descriptive study of 243 students. Dental caries' prevalence was evaluated by DMFT and ceo-d index; caries severity was measured by using ICDAS II 2005 criteria and family variables were ascertained by filling out a questionnaire that included the Family APGAR (adaptation, partnership, growth, affection and resolve) for measuring family functioning. Descriptive statistics were used 
when analysing relationships and the Chi-square test was used for variables. Results Caries' prevalence was $51 \%(45-9559 \% \mathrm{Cl})$. It was shown that $38 \%(31-44$ $95 \% \mathrm{Cl}$ ) of parents had reached secondary school (but not completed it), $44 \%$ (39-54 $95 \% \mathrm{Cl})$ were living with a partner, $47 \%(40-5395 \% \mathrm{Cl})$ had low socioeconomic status, $53 \%(47-5795 \% \mathrm{Cl})$ were living in nuclear families, $47 \%(41-5395 \% \mathrm{Cl})$ were receiving less than minimum wage income, $66 \%(58-7095 \% \mathrm{Cl})$ were affiliated to the government health system and $59 \%(52-6695 \% \mathrm{Cl})$ were living in dysfunctional families. Bivariate analysis only revealed statistical significance regarding the fathers' incomplete secondary schooling and the presence of dental caries $(p=0.04)$.

Conclusions Even though most variables showed no statistical significance regarding explaining the presence of dental caries, they did provide epidemiologically important indicators which would facilitate decisions being made from a public health standpoint.

Key Words: Dental caries, family practice, preschool (source: Mesh, NLM).

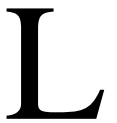

a caries dental se ha considerado como la enfermedad de mayor peso en la historia de la morbilidad bucal a nivel mundial. En la actualidad, su aparición se asocia en gran manera con factores socioculturales, económicos, del ambiente y del comportamiento. Afecta entre el $60 \%$ y $90 \%$ de la población escolar según la OMS (1). La caries dental es un proceso multifactorial, al estudiar la temática se abordan por lo general factores de riesgo biológicos y se pasa por alto el hecho de que la familia y en especial los padres y/o cuidadores forman una parte esencial de futuros programas de promoción y prevención para poder disminuir la morbilidad, el progreso de la enfermedad y los costos de la misma.

Factores presentes en las familias como la disfuncionalidad familiar y el tipo de estructura familiar, como el aumento en la actualidad de la familia monoparental, podrían incrementar el riesgo de presentar caries dental y acentuar su severidad (2). Actualmente poco se ha estudiado al respecto a nivel de la población escolar y tampoco se ha logrado explicar la relación que existe entre estos factores y la caries dental, lo que conlleva a que no se puedan tomar medidas desde la institución universitaria docente, asistencial e investigativa que contribuyan a controlar o evitar que estos factores se presenten desde el diseño de los programas de promoción y prevención implementados. Desde esta perspectiva, hoy es claro que la salud oral no es solo problema del odontólogo; la familia y la comunidad juegan también un papel importante en la promoción de la salud bucal y la prevención de enfermedades.

El objetivo de este estudio fue estimar la prevalencia y severidad de la caries dental en niños de la institución educativa John F. Kennedy de la ciudad de Cartagena y su relación con factores familiares. 


\section{MÉTODOS}

Estudio descriptivo transversal, en la institución educativa oficial John F. Kennedy de la ciudad de Cartagena. La población marco la constituyen 661 niños que cursan preescolar y básica primaria. El tamaño de la muestra fue de 243 sujetos, calculado a partir de una prevalencia esperada de caries dental del $55 \%$ (3), nivel de confianza del $95 \%$, error permitido de 0,05 , y un poder del $80 \%$. El proceso de selección se realizó en forma probabilística estratificada, a partir de una fijación proporcional por el grado de escolaridad de los niños. Como criterios de exclusión se tuvieron en cuenta las enfermedades sistémicas como diabetes, hipertensión, síndrome de Down o cualquier tipo de discapacidad motora y sensorial que pudieran sobreestimar la ocurrencia tanto de la caries dental como de los factores explicativos.

Para el inicio de las mediciones se solicitó el consentimiento de la institución educativa y a los padres de los niños, diligenciándose en los padres un consentimiento informado por escrito, teniendo en cuenta la resolución 8430 de 1993 del Ministerio de Salud de la República de Colombia.

Se utilizó un instrumento clínico, basado en el formato Guías de práctica clínica en salud oral de la Secretaria Distrital de Salud, Bogotá- ACFO, 2006, aplicándose el índice de COP para la prevalencia de experiencia de caries, tomando como parámetro un valor igual o mayor a dos. Para la severidad de Caries se utilizaron los criterios diagnósticos clínicos ICDAS II 2005 (4). Este diagnóstico se llevó a cabo en los consultorios odontológico ubicados al interior del colegio. Posteriormente, se realizaron visitas domiciliarias para la medición de las características, estructura y funcionalidad familiar, utilizando un cuestionario con 32 preguntas estructuradas para una sola opción de respuesta, dirigido a los padres de los niños seleccionados. Para obtener la validez de apariencia y la plausibilidad teórica del instrumento, se realizaron dos sesiones de estandarización, por los especialistas en Salud Familiar y Odontopediatría de la institución universitaria. Para los resultados de las pruebas de concordancia entre los examinadores clínicos, se realizó un análisis intra e inter-examinador, asumiendo un grado de acuerdo de 0,81, a través de la prueba Kappa Cohen. Para el procesamiento y análisis de la información se utilizaron los programas Microsoft Excel 2007®, EPIINFO versión 3.5.1® y STATA X® licencia No: 1990540768. Las medidas descriptivas se estimaron a partir de ocurrencias de presencia y severidad de la caries dental y las variables familiares y demográficas, asumiendo intervalos de confianza del $95 \%$. La relación se evaluó mediante un análisis bivariado, aplicándose la prueba $\chi^{2}$ para poner a prueba la significancia estadística. 


\section{RESULTADOS}

La Tabla 1 muestra la distribución de las características de la población objeto de estudio.

Tabla 1. Población objeto por edad, sexo y escolaridad en la Institución Educativa John F. Kennedy de la ciudad de Cartagena. 2009

\begin{tabular}{|c|c|c|c|c|c|c|}
\hline \multirow[b]{2}{*}{ Variables } & \multicolumn{2}{|c|}{ Total $n=243$} & \multicolumn{2}{|c|}{ Niños $n=123$} & \multicolumn{2}{|c|}{ Niñas $n=120$} \\
\hline & Número & $\%$ & Número & $\%$ & Número & $\%$ \\
\hline \multicolumn{7}{|l|}{ Rango Edad } \\
\hline 4 a 5 & 41 & 17 & 18 & 15 & 23 & 19 \\
\hline 6 a 7 & 66 & 27 & 28 & 23 & 38 & 32 \\
\hline 8 a 9 & 74 & 30 & 38 & 31 & 36 & 30 \\
\hline 10 a 11 & 55 & 23 & 36 & 29 & 19 & 16 \\
\hline 12 a 13 & 7 & 3 & 3 & 2 & 4 & 3 \\
\hline \multicolumn{7}{|l|}{ Escolaridad } \\
\hline Transición & 35 & 14 & 16 & 13 & 19 & 16 \\
\hline Primero & 35 & 14 & 19 & 15 & 17 & 14 \\
\hline Segundo & 28 & 12 & 13 & 11 & 14 & 12 \\
\hline Tercero & 47 & 19 & 20 & 16 & 27 & 23 \\
\hline Cuarto & 47 & 19 & 23 & 19 & 24 & 20 \\
\hline Quinto & 51 & 21 & 32 & 26 & 19 & 16 \\
\hline Total & 243 & 100 & 123 & 100 & 120 & 100 \\
\hline
\end{tabular}

Tabla 2. Prevalencia de los Factores Familiares en Niños de la Institución Educativa John F. Kennedy. Cartagena 2009

\begin{tabular}{|c|c|c|c|c|}
\hline Variables Familiares & $\begin{array}{c}\text { Frecuencia } \\
n=243\end{array}$ & Porcentaje (\%) & \multicolumn{2}{|c|}{$\mathrm{IC}=95 \%$} \\
\hline Familia Extensa & 92 & 38 & 31 & 44 \\
\hline Familia Nuclear & 130 & 53 & 47 & 57 \\
\hline Dos hijos en la familia & 100 & 41 & 34 & 50 \\
\hline Parejas en Unión Libre & 112 & 46 & 39 & 53 \\
\hline Casa como tipo de vivienda & 170 & 70 & 64 & 75 \\
\hline Vivienda no propia & 90 & 37 & 30 & 43 \\
\hline Ambos padres ejercen autoridad & 85 & 35 & 28 & 41 \\
\hline $\begin{array}{l}\text { Rol del padre formador de hijos y proveedor } \\
\text { económico }\end{array}$ & 133 & 55 & 48 & 61 \\
\hline Horario laboral de la madre igual a ocho horas & 52 & 21 & 16 & 26 \\
\hline Padres cuidadores del niño & 154 & 63 & 57 & 69 \\
\hline Abuelos cuidadores del niño & 56 & 23 & 17 & 28 \\
\hline Ingresos económicos hasta un SMMLV & 115 & 47 & 41 & 54 \\
\hline $\begin{array}{l}\text { Afiliación al Sistema de Salud a través del } \\
\text { Régimen Subsidiado }\end{array}$ & 157 & 65 & 59 & 70 \\
\hline Antecedentes familiares de Hipertensión & 53 & 22 & 16 & 27 \\
\hline Muerte de menores de cinco años & 5 & 2 & 0,10 & 14 \\
\hline Familia presenta problemas de salud oral & 160 & 66 & 60 & 71 \\
\hline Visitan al odontólogo por Curación & 85 & 35 & 29 & 41 \\
\hline Visitan al odontólogo por Urgencia & 49 & 20 & 15 & 25 \\
\hline Disfuncionalidad familiar leve & 94 & 39 & 33 & 45 \\
\hline Disfuncionalidad familiar moderada & 38 & 16 & 11 & 20 \\
\hline Disfuncionalidad Familiar severa & 5 & 2 & 0,10 & 14 \\
\hline
\end{tabular}


Prevalencia y severidad de caries dental. Se encontró una prevalencia de caries dental de $51 \%$ (IC $95 \%$; 45-59), siendo mayor para los niños entre 8 y 9 años en un $32 \%(\mathrm{p}=0.04)$. De acuerdo a los criterios de severidad ICDAS 2005, la lesión encontrada con mayor frecuencia fue la tipo 3 en un $2 \%$ (IC $95 \% ; 2,01-2,72$ ). El tipo de superficie dentaria más comprometida fue la oclusal en un $40 \%$ (IC $95 \%$; 2,75-3,52), seguida de la vestibular con el $38 \%$ (IC $95 \% ; 2,77-3,41$ ). En cuanto al sexo, se observó la mayor frecuencia en las niñas con $56 \%(\mathrm{p}=0,02)$.

Prevalencia de los factores socio-demográficos y familiares. De las variables socio demográficas las de mayor prevalencia fueron el estrato socioeconómico de nivel dos (47 \%; IC $95 \%$; 40-53), grado de escolaridad en el padre de secundaria incompleta en un $38 \%$ ( IC 95 \%; 31-44) y la unión libre en un $46 \%$ (IC $95 \%$; 39-53). De las variables familiares se encontró la familia nuclear en un $53 \%$ (IC $95 \%$; 47-57), familias con ingresos económicos hasta un salario mínimo en un $47 \%$ (IC 95\%;41-53), $66 \%$ (IC $95 \%$; 59-73) de las familias estaban afiliadas al sistema de salud, el $66 \%$ (IC $95 \%$; 60-73) presentan problemas de salud bucal, el $35 \%$ (IC $95 \%$; 4852) visitan al odontólogo por curación y el $59 \%$ (IC $95 \%$; 52-66) viven en familias disfuncionales (Tabla 2).

Tabla 3. Descripciones de las variables socio demográficas y familiares relacionadas con la presencia o no de caries dental de los escolares. Cartagena 2009

\begin{tabular}{|c|c|c|c|c|c|c|}
\hline \multirow[t]{2}{*}{ Variables } & \multicolumn{2}{|c|}{ Caries Dental } & \multicolumn{2}{|c|}{ Sin Caries dental } & \multirow[b]{2}{*}{$\mathrm{Chi}^{2}$} & \multirow[b]{2}{*}{$p$} \\
\hline & Frecuencia & $\%$ & Frecuencia & $\%$ & & \\
\hline Edad entre 8 y 9 años & 40 & 32 & 34 & 29 & 10,03 & $0,04^{*}$ \\
\hline Sexo femenino & 70 & 56 & 50 & 42 & 5,06 & $0,02^{*}$ \\
\hline Estrato Socioeconómico II & 56 & 45 & 57 & 48 & 0,34 & 0,95 \\
\hline Secundaria incompleta de padres & 35 & 28 & 57 & 48 & 13,22 & $0,04^{*}$ \\
\hline Secundaria incompleta de madres & 46 & 37 & 52 & 44 & 4,46 & 0,61 \\
\hline Parejas en unión libre & 55 & 44 & 57 & 48 & 8,3 & 0,217 \\
\hline Dos hijos en las familias & 47 & 38 & 53 & 45 & 5,71 & 0,57 \\
\hline Familia Extensa & 48 & 39 & 44 & 37 & 0,07 & 0,7 \\
\hline Familia Nuclear & 64 & 52 & 66 & 37 & 0.36 & 0.54 \\
\hline Ingresos hasta un SMMLV & 52 & 42 & 63 & 53 & 3,58 & 0,46 \\
\hline Afiliación SGSS régimen subsidiado & 82 & 66 & 75 & 63 & 2,72 & 0,74 \\
\hline Hombre formador de hijos y proveedor & 64 & 52 & 69 & 58 & 5,99 & 0,11 \\
\hline Horario laboral Madre hasta ocho horas & 32 & 26 & 20 & 17 & 9,79 & 0,13 \\
\hline Antecedentes familiares hipertensión & 30 & 24 & 23 & 19 & 18,05 & 0,2 \\
\hline Familias con problemas de salud oral & 82 & 66 & 78 & 66 & 0,009 & 0,9 \\
\hline Visitan al odontólogo por curación & 48 & 39 & 37 & 31 & 1,55 & 0,2 \\
\hline Visitan al odontólogo por urgencia & 23 & 19 & 26 & 22 & 0,41 & 0,52 \\
\hline Disfuncionalidad familiar leve & 51 & 41 & 43 & 36 & 0,63 & 0,42 \\
\hline Disfuncionalidad familiar moderada & 20 & 16 & 18 & 15 & 0,04 & 0,83 \\
\hline Disfuncionalidad familiar severa & 2 & 2 & 3 & 3 & 0,24 & 0,61 \\
\hline
\end{tabular}

* Estadísticamente significativo 
Relación entre los factores socio-demográficos y familiares y la prevalencia de experiencia de caries dental. Sólo se encontró significancia estadística al relacionar el nivel de escolaridad de secundaria incompleta del padre con la presencia de caries dental $(\mathrm{p}=0,04)$. En general se encontraron grandes coincidencias en la prevalencia de caries dental con la presencia de aquellos factores socios demográficos y familiares de mayor ocurrencia reportados anteriormente pero sin ser estadísticamente significativas (Tabla 3).

\section{DISCUSIÓN}

Es interesante observar que a pesar de las actividades en promoción y prevención en salud oral, aún se encuentren altas prevalencias de caries dental en la población objeto de estudio. Estos resultados son muy similares a los encontrados por González (5) quien en niños de hogares infantiles del Instituto Colombiano de Bienestar Familiar en la ciudad de Cartagena, reportó prevalencia de $55 \%$. Cabe resaltar que en ambos estudios se utilizaron criterios de diagnósticos de caries ICDAS 2005 para evaluar la severidad de la Caries. Por otro lado, en cuanto al sexo, la mayor frecuencia fue en mujeres con $56 \%$, similar a los resultados encontrados por Medina (6) en niños de hogares infantiles en México.

Con relación al tipo de superficie dentaria más comprometida, en el presente estudio se observó la oclusal, esto es explicable debido a que la profundidad de la fisura favorece la acumulación de la placa sobre la morfología oclusal, sumando a esto, el tipo de bacteria predominante en dicha superficies pueden potenciar las tendencias de caries activas, resultados similares a los reportados por Sánchez (7) en un estudio en México sobre factores de riesgo a caries en escolares. En cuanto a la severidad de las lesiones de caries, las lesiones microcavitacionales tipo 3 según ICDAS II 2005, fueron las más frecuentes, lo que indica una mayor severidad de las lesiones por pérdida superficial de compromiso estructural, este hallazgo permite planear más actividades de operatorias, más costos en los servicios de salud oral sin mencionar más estrés en el paciente.

Smilkstein manifiesta que la influencia de la familia en el proceso saludenfermedad es de vital importancia, ya que el grupo familiar puede funcionar como fuente de salud o enfermedad, o viceversa, y el paciente puede llegar a comprometer la salud emocional de los individuos que componen la familia $(8,9)$. Igualmente la familia puede influir en el proceso salud enfermedad de la Caries dental (10). Uno de los factores familiares analizados en el presente estudio fue el nivel socioeconómico. Reisine (11) encontró que la 
prevalencia de caries aumenta conforme disminuye el nivel socioeconómico. En este estudio, los niños que presentaban caries dental viven en su mayoría en estrato socioeconómico bajo nivel II, y sus familias reciben ingresos menores de un salario mínimo legal vigente, resultados similares a los encontrados por Montellano (12), en el cual el $72 \%$ de las familias recibían 1 SMMLV. Es claro suponer que las familias con estas condiciones no dispondrían de medios o recursos suficientes para invertir en su salud oral.

Por otro lado, el nivel de escolaridad bajo de las madres ha estado relacionado con la aparición de caries dental (13-15). Es importante también conocer el nivel de escolaridad de los cuidadores cuando no son los padres y su relación con la aparición de caries dental. En el presente estudio se encontró que en los niños que presentaban caries dental, el nivel de escolaridad de los padres más frecuente fue la secundaria incompleta y cuando los cuidadores eran los abuelos se observó un nivel de escolaridad bajo correspondiente a primaria.

Con respecto a la afiliación de las familias al sistema de salud, se ha considerado como riesgo no tener acceso a los servicios de salud por falta de afiliación o por no uso de los servicios $(16,17)$. Es contradictorio encontrar en los resultados que un gran porcentaje de los niños con caries dental, se encontraban afiliados al sistema de seguridad social en salud en el régimen subsidiado. Varias consideraciones pueden surgir para explicar este resultado, en primera instancia puede darse la escasa utilización de los servicios, ya sea por dificultad de acceso o mala calidad de los mismos, además se pueden presentar pocas oportunidades para acceder a las actividades de promoción y prevención en salud bucal.

Según los resultados del presente estudio en las familias nucleares y conformadas por cuatro miembros, los niños presentaban la mayor frecuencia de caries dental. También se encontró un gran porcentaje de niños con caries dental donde principalmente eran cuidados por el padre, lo que nos indica que esta figura paterna no se encuentra bien educada en promoción y prevención en salud bucal. En la literatura la estructura familiar o tipo de familia y el vivir con los padres, se ha relacionado con la presencia de Caries dental (2). En este sentido, Pau encontró que los niños que vivían con uno de sus dos padres presentaban mayor prevalencia de dolor dental, en este misma perspectiva indicaron resultados reportados por González (18) en la ciudad de Cartagena, pero en cuanto a la presencia de la caries dental. 
Con respecto a los roles ejercidos por los padres en la crianza de los hijos Arenas (19) refiere que la pobreza obliga a la mujer a mantenerse empleada y fuera del hogar descuidando sus otras labores como madre y ama de casa. Los padres de la población en estudio permanecen hasta ocho horas por fuera del hogar. Al estar las madres tan ocupadas se delegaría la responsabilidad de cuidar a los niños a otras personas como los abuelos como fue expuesto por Franco (12) en un estudio realizado con madres y niños de la ciudad de Medellín, esto demuestra la importancia de incluir a los cuidadores diferentes a los padres en las actividades de promoción y prevención en salud bucal.

De los niños con caries dental, un gran porcentaje viven en familias que presentan problemas de salud oral y visitan al Odontólogo por curación. Se observa aquí la percepción e importancia de las familias hacia el tratamiento, transmitiéndose esta percepción en los niños, aspecto este que podría actuar como un factor de riesgo para adquirir la caries dental (20).

A partir de los resultados obtenidos se concluye que aunque la mayoría de las variables de salud familiar si bien no mostraron significancia estadística para explicar la presencia de la caries dental en esta población, nos aportan indicadores de importancia epidemiológica, lo cual permitiría tomar decisiones desde el punto de vista de salud pública

Agradecimientos. A la Universidad de Cartagena por el apoyo académico y administrativo durante la realización de este estudio. A la Institución educativa Jhon F. Kennedy de la ciudad de Cartagena, por el apoyo logístico durante las actividades realizadas dentro del plantel.

\section{REFERENCIAS}

1. Higashida B. Odontología preventiva. Primera Edición. México: McGraw-Hill; 2000.

2. Pau A, Khan S, Babar M, Croucher R. Dental pain and care-seeking in 11-14-yr-old adolescents in a low-income country. Europe Journal Oral Science 2008; 116(5):451-7.

3. González F, Alfaro L, Nieto C, Carmona L. Evaluación de las condiciones de salud oral y los conocimientos en niños escolares entre 7 y 14 años de la población de la BoquillaCartagena de indias, 2005. Revista Científica Facultad de Odontología Universidad El Bosque 2006; 12: 25 - 34.

4. Ismail AI, Sohn W, Tellez M, Willem M, Bertz J, Lepkowski. Risk indicators for dental Caries using the International Caries Detection and Assessment System (ICDAS). Community Dent Oral Epidemiol 2008; 36:55-68.

5. González F, Luna L, Solana M. Relación entre caries dental y factores de riesgo en niños de hogares infantiles del Bienestar familiar de Cartagena. Rev Ustasalud 2008; 7:87-95. 
6. Segovia-Villanueva A, Estrella R, Medina C, Maupome G. Severidad de caries y factores asociados en preescolares de 3-6 años de edad en Campeche, México. Rev. salud pública (Bogotá). 2005;7: 56-69.

7. Sánchez-Pérez L, Méndez I, Sáenz L. Línea basal de factores de riesgo a caries en escolares. Bol. Med. Hosp. Infant. Mex. 2005; 62: 33-44.

8. Minuchin S. Familia y Terapia familiar. Argentina: Editorial Gedisa; 2001.

9. Smilkstein G. The Family Apgar: A proposal for a family function test and its use by physicians. Journal family practice. 1978; 6:1231-9.

10. Fejerskov $\mathrm{O}$. Changing paradigms in concepts on dental caries: consequences for oral health care. Caries Research 2004; 38(3):182-91.

11. Reisine ST, Psoter W. Socioeconomic Status and Selected behavioral determinants as risk factors for dental caries. Journal of Dental Education 2001; 65(10): 1009-16.

12. Montellano G, Brieke W, Cáceres S, Orduña C, Serrano S. Factores de riesgo en pacientes con caries temprana de la infancia del departamento de estomatología del hospital para el niño poblano. Revista Oral 2004; 5:230-232.

13. Franco A, Santamaría A, Kurzer E, Castro L, Giraldo M. El menor de seis años: situación de caries y conocimientos y prácticas de cuidado bucal de sus madres. Revista Ces Odontología 2004; 17(1):19-29.

14. Bustamante Z, Camargo L. Estado de salud bucal de niños preescolares y escolares de nivel socioeconómico alto y medio alto. Revista Ces Odontología 1998; 11(1): 3538.

15. Ferreira SH, Béria JU, Kramer PF, Feldens EG, Feldens CA. Dental caries in 0- to 5-yearold Brazilian children: prevalence, severity, and associated factors. Int $\mathrm{J}$ Paediatr Dent. 2007;17(4):289-96.

16. Amaya P. Instrumento de Riesgo Familiar Total. Manual. Aspectos teóricos, psicométricos de estandarización y de aplicación. RFT: 5-33 (Tercer tomo). Santa Fé de Bogotá: Editorial Universidad Nacional de Colombia; 2004.

17. Agudelo A, Zapata D, Martínez E. Dinámica familiar y el fomento de la salud bucal en poblaciones desplazadas y destechadas. Revista Ces Odontología 2007; 20(2): 23-30.

18. González F, Sánchez R, Carmona L. Indicadores de riesgo para la caries dental en niños preescolares de La Boquilla, Cartagena. Rev Salud Pública (Bogotá). 2009; 11(4): 620-630.

19. Arenas A. Consideraciones en torno a la jefatura de hogar femenino en población urbana de menores recursos. Reflexiones para la intervención en la problemática familiar. Consejería Presidencial para la Política Social. PNUD. Santafé de Bogotá; 1995.

20. Cuartas J, Alvear A. Relación entre percepción de los padres sobre el tratamiento odontológico y sus hábitos de higiene oral, con la historia de caries dental en sus hijos, entre 3 y 5 años de edad. Revista Ces odontología 2002; 15(1):13-18. 